\title{
AKT2 is involved in the IL-17A-mediated promotion of differentiation and calcification of murine preosteoblastic MC3T3-E1 cells
}

\author{
JING-YI TAN ${ }^{1}$, LI-HONG LEI ${ }^{1}$, XIAO-TAO CHEN ${ }^{2}$, PEI-HUI DING ${ }^{1}$, YAN-MIN WU $^{1}$ and LI-LI CHEN ${ }^{1}$ \\ ${ }^{1}$ Department of Oral Medicine, The Second Affiliated Hospital, School of Medicine, Zhejiang \\ University, Hangzhou, Zhejiang 310009; ${ }^{2}$ Department of Oral Medicine, People's Hospital \\ of Xinjiang Uygur Autonomous Region, Urumqi, Xinjiang 830001, P.R. China
}

Received November 10, 2016; Accepted June 27, 2017

DOI: $10.3892 / \mathrm{mmr} .2017 .7315$

\begin{abstract}
Interleukin (IL)-17A exhibits pleiotropic biological activities and serves a role in the progression of periodontitis. However, data describing the association between IL-17 and osteogenesis are not conclusive. It was previously demonstrated that RAC- $\beta$ serine/threonine protein kinase (AKT2)-specific knockdown in MC3T3-E1 cells weakened osteogenic effects. The role of AKT2 in the regulation of IL-17A for osteoblast differentiation and calcification remains unclear. The MTT method was adopted in the present study to assess cell proliferation; cell cycle distribution was analyzed by flow cytometry. Following osteogenic induction treatment, the involvement of phosphatidylinositol 3-kinase (PI3K) and phosphorylated-PI3K was evaluated by western blotting. The effects of IL-17A on osteogenesis-associated markers, including Runt-related transcription factor 2 (Runx-2), alkaline phosphatase (ALP) and osteocalcin (OCN) were evaluated by reverse transcription-quantitative polymerase chain reaction (RT-qPCR) analysis. An ALP activity assay and Alizarin Red $\mathrm{S}$ staining were used to assess the differentiation and calcification functions. AKT2 knockdown inhibited MC3T3-E1 cell proliferation, inducing significantly increased G0/G1 cell counts, and reduced S and G2/M cell numbers. IL-17A exerted no significant effects. The protein levels of $\mathrm{p}-\mathrm{PI} 3 \mathrm{~K}$, gene expression levels of IL-17A, Runx-2, ALP and OCN, and relative ALP activity and calcification areas were increased in the induction group, and these effects were markedly promoted by treatment with IL-17A. AKT2 knockdown in MC3T3-E1 cells resulted in reduced IL-17A-induced differentiation and
\end{abstract}

Correspondence to: Professor Li-Li Chen, Department of Oral Medicine, The Second Affiliated Hospital, School of Medicine, Zhejiang University, 88 Jiefang Road, Hangzhou, Zhejiang 310009, P.R. China

E-mail: chenlili_1030@163.com

Key words: interleukin-17A, RAC- $\beta$ serine/threonine protein kinase, osteoblast differentiation, osteoblast calcification calcification, although it was not completely inhibited. The results of the present study suggested that AKT2 signaling was required for MC3T3-E1 cell proliferation. IL-17A promoted osteoblast differentiation and calcification in a partly AKT2-dependent manner in MC3T3-E1 cells in vitro, possibly reflecting compensation by other signaling pathways. The results of the present study may offer novel perspectives to guide the clinical strategy for the prevention and treatment of periodontitis.

\section{Introduction}

Periodontitis is a chronic inflammatory disease that destroys the periodontal ligament and alveolar bone, and constitutes a major cause of tooth loss in adults $(1,2)$. It is caused by bacterial plaque, which leads to impaired immune and inflammatory responses (1). Currently, investigations have primarily focused on defining the complex regulatory interactions between the immune system and bone remodeling, which is widely known as 'osteoimmunology' (3).

Mounting evidence indicates that the novel $\mathrm{CD}^{+} \mathrm{T}$-cell subset termed 'Th17' and the signature pro-inflammatory cytokine interleukin (IL)-17 have a potentially important role in gingival inflammation and subsequent bone destruction in the pathogenesis of periodontitis (4). Recent clinical studies reported that the proportion of Th17 cells in the peripheral blood, plasma IL-17 levels and IL-17 expression in gingival lesions are significantly increased in patients with chronic periodontitis compared with healthy controls $(5,6)$; da Costa et al (7) additionally observed increased accumulation of $\mathrm{IL}-17^{+}$and tumor necrosis factor-related activation protein $^{+}$cells in periodontal lesions, indicating that high numbers of osteoclasts in local tissues may be associated with the presence of $\mathrm{IL}-17^{+}$cells.

By contrast, Yu et al (8) demonstrated that IL-17 exerts a profound bone-protective effect on bone loss in periodontal disease via IL-17 receptor A (IL-17RA) signaling. A recent study reported that the Tannerella forsythia wecC deletion mutant TFM-ED1 increases the Th17 response without enhancing osteoclastic activity, suggesting a protective role for Th17/IL-17 in the pathogenesis of periodontitis (9). A previous 
study indicated that osteogenic cells may be responsive to IL-17, and IL-17 modulates osteoclast activity (10). However, the role of IL-17A in bone protection is poorly understood.

In a previous study, it was demonstrated that RAC- $\beta$ serine/threonine protein kinase (AKT2) knockdown weakened the osteogenic effects of preosteoblastic MC3T3-E1 cells, with reduced osteocalcin $(\mathrm{OCN})$ expression and calcified deposits (11). Mukherjee et al (12) reported that AKT2 promoted bone morphogenetic protein 2-mediated osteoblast differentiation. RAC- $\alpha$ serine/threonine protein kinase (AKT) is activated by phosphatidylinositol 3-kinase (PI3K), resulting in the phosphorylation of other host proteins that affect cell proliferation, growth, the cell cycle and survival $(13,14)$. Furthermore, a complex relationship exists between IL-17 and PI3K/AKT signaling, which triggers multiple actions: IL-17A regulation in stimulated T-B cell co-culture is preferentially associated with the PI3K pathway (15); IL-17-producing natural killer T cells are essential for homeostasis and survival via PI3K/AKT signaling (16); and Porphyromonas gingivalis (a periodontopathogen) lipopolysaccharide is involved in periodontal disease-induced bone destruction and may mediate IL-17 and IL-23 release from human periodontal ligament cells, with PI3K/AKT signaling serving a role in this process (17). However, it remains unclear whether the PI3K/AKT pathway may be activated by IL-17A in the process of osteogenesis. In addition, no reports assessing the involvement of AKT2 in osteoblast differentiation and calcification in association with IL-17A have been published.

Therefore, the purpose of the present study was to examine the effects of IL-17A on the proliferation, differentiation and calcification of preosteoblastic MC3T3-E1 cells and to examine the associated signaling pathways. In a previous study, AKT2 knockdown $\left(\mathrm{AKT}^{-/}\right.$) cells were obtained by RNA interference (RNAi) following transfection with an effective AKT2-specific RNAi plasmid (11). The present study further investigated whether AKT2 was implicated in IL-17A-mediated osteoblast differentiation and calcification by examining cell proliferation in addition to the expression of early and late osteogenic markers. The results of the present study provided novel insights regarding the role of AKT2 in IL-17A-mediated osteogenesis and may help elucidate the mechanism of bone destruction in periodontitis.

\section{Materials and methods}

Materials. Mouse IL-17A was from Peprotech Inc. (Rocky Hill, NJ, USA). Dexamethasone, L-ascorbic acid, $\beta$-glycerophosphate, and dimethyl sulfoxide (DMSO) were purchased from Sigma-Aldrich (Merck KGaA, Darmstadt, Germany). All cell culture media and supplements were from Gibco (Thermo Fisher Scientific, Inc., Waltham, MA, USA). Reagents for the reverse transcription-quantitative polymerase chain reaction (RT-qPCR) were obtained from Takara Bio, Inc. (Otsu, Japan). MTT was purchased from Amresco, LLC (Solon, OH, USA). Rabbit anti-PI3K (cat. no. 4292), anti-phosphorylated (p)-PI3K (cat. no. 4228) and anti-GAPDH (cat. no. 2118) monoclonal antibodies were purchased from Cell Signaling Technology, Inc. (Danvers, MA, USA). Goat anti-rabbit immunoglobulin $\mathrm{G}$ secondary antibodies (cat. no. BA1054) were obtained from Wuhan Boster Biological
Technology, Ltd. (Wuhan, China). Enhanced chemiluminescence (ECL) detection reagent was purchased from Thermo Fisher Scientific, Inc. Alkaline phosphatase (ALP) activity kit was provided by Beyotime Institute of Biotechnology (Haimen, China).

Cell culture. MC3T3-EI cells, a murine calvarial preosteoblastic cell line with the capacity to differentiate into osteoblasts and deposit minerals in vitro (18), were acquired from the Type Culture Collection of the Chinese Academy of Sciences (Shanghai, China), and maintained in $\alpha$-Minimum Essential Medium containing 10\% fetal bovine serum and $1 \%$ penicillin-streptomycin (normal medium, NM) at $37^{\circ} \mathrm{C}$ under $5 \%(\mathrm{v} / \mathrm{v}) \mathrm{CO}_{2}$ in a humidified atmosphere. For osteogenic induction, MC3T3-E1 cells were incubated in osteogenic induction medium (Ind.) containing $10 \mathrm{nM}$ dexamethasone, $50 \mu \mathrm{g} / \mathrm{ml} \mathrm{L}$-ascorbic acid and $10 \mathrm{mM} \beta$-glycerophosphate. IL-17A was tested at a concentration of $50 \mathrm{ng} / \mathrm{ml}$, with the medium being replaced every $48 \mathrm{~h}$.

AKT2 knockdown by RNAi in MC3T3-E1 cells. The knockdown of AKT2 in MC3T3-E1 cells was described in detail in a previous report (11).

Cell proliferation assay. Cells were plated at $1 \times 10^{3}$ cells/well in 96-well plates in serum-free medium for $24 \mathrm{~h}$. In order to assess the effects of IL-17A on cell proliferation, the control (wild type MC3T3-E1 cells) and SAKT2 (AKT2 ${ }^{-/-}$MC3T3-E1 cells) groups were incubated in NM with or without IL-17A for 24, 48, 72 and 96 h, respectively. Cell viability was assessed using an MTT assay, according to the manufacturer's protocol. A total of $50 \mu \mathrm{l}$ MTT $(5 \mathrm{mg} / \mathrm{ml})$ was added to each well post-treatment. After $4 \mathrm{~h}$ of incubation in a $5 \% \mathrm{CO}_{2}$ incubator at $37^{\circ} \mathrm{C}$, the culture medium was carefully aspirated, and $150 \mu \mathrm{l}$ DMSO added to each well. Absorbance was read at $490 \mathrm{~nm}$ using a spectrophotometer (Bio-Rad Laboratories, Inc., Hercules, CA, USA).

Cell cycle analysis. Cells were inoculated at $5 \times 10^{4}$ cells/well in 6-well plates, and starved in serum-free medium for $24 \mathrm{~h}$. Following treatment with $50 \mathrm{ng} / \mathrm{ml}$ IL-17A in NM and incubation at $37^{\circ} \mathrm{C}$ for $48 \mathrm{~h}$, the cells were resuspended to $\sim 10^{6}$ cell $/ \mathrm{ml}$ in PBS. Subsequently, $1 \mathrm{ml}$ cell suspension was transferred to $3 \mathrm{ml} \mathrm{70 \%} \mathrm{ethanol} \mathrm{by} \mathrm{slowly} \mathrm{pipetting,} \mathrm{vortexed} \mathrm{at} \mathrm{full} \mathrm{speed,}$ and incubated at $4^{\circ} \mathrm{C}$ overnight. Following centrifugation at $1,200 \mathrm{x} \mathrm{g}$ for $5 \mathrm{~min}$ at room temperature, the cell pellets were resuspended in $2 \mathrm{ml}$ PBS and rehydrated for $15 \mathrm{~min}$, and mixed with $1 \mathrm{ml}$ cell cycle staining solution containing propidium ioidide [Multi Sciences (Lianke) Biotech Co., Ltd., Hangzhou, China]. Cell cycle distribution was analyzed by flow cytometry (BD Biosciences, Franklin Lakes, NJ, USA) following $30 \mathrm{~min}$ of incubation at room temperature. Data were analyzed using CellQuest Pro software, version 5.1 (BD Biosciences).

Western blotting. Control and SAKT2 group cells were cultured in NM and Ind., separately. Cells in Ind. were treated with or without IL-17A, and the grouping was the same in the following experiments. A total of $48 \mathrm{~h}$ following incubation of $5 \times 10^{4}$ cells/well in 6-well plates, radioimmunoprecipitation assay cell lysate buffer (Beyotime Institute of Biotechnology) 
was used for cell lysis. Equal amounts of total protein $(60 \mu \mathrm{g})$, as quantified by a bicinchoninic acid kit (Thermo Fisher Scientific, Inc.), were resolved by SDS-PAGE on a $10 \%$ gel and transblotted onto a polyvinylidene fluoride membrane (EMD Millipore, Billerica, MA, USA). Subsequently, the membranes were blocked with $5 \%$ non-fat milk for $2 \mathrm{~h}$ at room temperature. Anti-p-PI3K (1:1,000), PI3K (1:1,000), and GAPDH $(1: 5,000)$ were incubated overnight at $4^{\circ} \mathrm{C}$. Membranes were washed and incubated with appropriate horseradish peroxidase-conjugated secondary antibodies $(1: 5,000)$ at room temperature for $1.5 \mathrm{~h}$. Following washing, immunoreactive bands were visualized using an ECL kit (Thermo Fisher Scientific, Inc.). Data were analyzed using Image J software, version 1.48 (National Institutes of Health, Bethesda, MD, USA).

RT-qPCR analysis. RT-qPCR was used to assess the gene expression of IL-17RA and osteogenic markers, including Runt-related transcription factor-2 (Runx-2), ALP, and osteocalcin (OCN). Cells were prepared as described above and total cellular RNA was isolated following treatment with TRIzol reagent (Invitrogen; Thermo Fisher Scientific, Inc.). A total of $1 \mu \mathrm{g}$ total RNA was reverse-transcribed to generate single-stranded cDNA using a PrimeScript ${ }^{\mathrm{TM}}$ RT Master Mix kit (Takara Bio, Inc.). The reverse transcription parameters were as follows: $37^{\circ} \mathrm{C}$ for $15 \mathrm{~min}, 85^{\circ} \mathrm{C}$ for $5 \mathrm{sec}$, and terminating at $4^{\circ} \mathrm{C}$. The expression levels of target genes were quantified with a SYBR ${ }^{\circledR}$ Premix Ex Taq ${ }^{\mathrm{TM}}$ II kit (Takara Bio, Inc.) on a StepOnePlus Real-Time PCR System (Applied Biosystems; Thermo Fisher Scientific, Inc.). The primer sequences were as follows: Forward, 5'-GCCCACTACCCT CACATCTT-3', and reverse, 5'-TCCTGTGCTCTCTCAAGT GC-3' for IL-17RA; forward, 5'-TTCTCCAACCCACGAATG CAC-3', and reverse, 5'-CAGGTACGTGTGGTAGTGAGT-3' for Runx-2; forward, 5'-TGACCTTCTCTCCTCCATCC-3', and reverse, 5'-CTTCCTGGGAGTCTCATCCT-3' for ALP; forward, 5'-TGCTTGTGACGAGCTATCAG-3', and reverse, 5'-GAGGACAGGGAGGATCAAGT-3' for OCN; and forward, 5'-GACTTCAACAGCAACTCCCAC-3', and reverse, 5'-TCC ACCACCCTGTTGCTGTA-3' for GAPDH. Amplification was performed for 40 cycles at $95^{\circ} \mathrm{C}(30 \mathrm{sec}), 95^{\circ} \mathrm{C}(5 \mathrm{sec})$, and $60^{\circ} \mathrm{C}$ (30 sec). Relative expression levels of target genes were derived by the relative quantitative method $\left(2^{-\Delta \Delta \mathrm{Cq}}\right)$ (19), and normalized to the mouse GAPDH gene.

ALP activity assay. To assess the early differentiation of MC3T3-E1 cells, ALP activity was analyzed using a specific kit, according to the manufacturer's protocol. Cells were lysed in lysis buffer (Beyotime Institute of Biotechnology) on day 14 . Subsequently, $50 \mu 1$ cell lysate was mixed with $50 \mu 1$ freshly prepared para-nitrophenylphosphate (pNPP) substrate and incubated at $37^{\circ} \mathrm{C}$ for $30 \mathrm{~min}$. The reaction was terminated by addition of $100 \mu \mathrm{l}$ stop solution, and the amounts of pNPP produced were quantified by measuring the absorbance at $405 \mathrm{~nm}$ on a spectrophotometer. ALP activity was normalized to the total protein concentration.

Analysis of cell calcification. For calcification assessment, MC3T3-E1 cells were cultured $\left(1 \times 10^{4}\right.$ cells/well in 6-well plates) under the same culture conditions as detailed above for 14 days. Alizarin Red staining of matrix mineralization was performed. Cells were fixed in $4 \%$ paraformaldehyde for $10 \mathrm{~min}$ at room temperature and stained with $0.1 \%$ Alizarin Red (Sigma-Aldrich; Merck KGaA)/Tris-HCl (pH 8.3) for $30 \mathrm{~min}$ at $37^{\circ} \mathrm{C}$, air-dried and photographed under a light microscope at x100 magnification (4 images/well; ZEISS Axio VertA1, Zeiss AG, Oberkochen, Germany). The calcified nodules were quantized by integrated optical density using Image Pro Plus 6.0 software (Media Cybernetics, Inc., Rockville, MD, USA).

Statistical analysis. Each experiment was performed in triplicate. Quantitative data are presented as the mean \pm standard deviation. Groups were compared using one-way analysis of variance, followed by the least significant difference post hoc test, using SPSS software (version 17.0; SPSS, Inc., Chicago, IL, USA). $\mathrm{P}<0.05$ was considered to indicate a statistically significant difference.

\section{Results}

Effects of IL-17A and AKT2 knockdown on MC3T3-E1 cell proliferation. A time-dependent assay was performed to assess the cell proliferation of wild type and AKT2 $2^{--}$MC3T3-E1 cells treated with or without IL-17A. At the $24 \mathrm{~h}$ time point, there was no statistically significant difference $(\mathrm{P}>0.05)$ among all 4 groups (Fig. 1A). However, progressive and significant increases were obtained at 48, 72 and 96 h. Notably, cell numbers were decreased upon $\mathrm{AKT} 2^{-/-}$treatment, regardless of stimulation with IL-17A ( $\mathrm{P}<0.05$; Fig. 1A). IL-17A elicited a similar effect, with a slight difference in proliferation, on wild type and AKT2 ${ }^{-/-}$cells $(\mathrm{P}>0.05$; Fig. 1A). For cell cycle distribution (Fig. 1B and C), AKT2 knockdown induced a significant increase in the number of cells arrested in G0/G1 $(\mathrm{P}<0.05)$, while reducing the numbers of $\mathrm{S}$ and $\mathrm{G} 2 / \mathrm{M}$ cells $(\mathrm{P}<0.05)$. IL-17A exerted no significant effect on $\mathrm{AKT}^{-/-}$cells compared with the control group $(\mathrm{P}>0.05)$.

IL-17A promotes differentiation and calcification in MC3T3-E1 cells. ALP and Runx-2 are important genes required for early osteoblast differentiation (20,21). As presented in Fig. 2, the levels of both genes were significantly increased in the induction group, while a more significant upregulation was detected at 7 days compared with 3 days. In addition, ALP and Runx-2 mRNA expression was markedly enhanced by IL-17A treatment $(\mathrm{P}<0.05$; Fig. $2 \mathrm{~A}$ and $\mathrm{B})$. OCN mRNA expression and Alizarin Red staining were quantified to assess osteoblast mineralization. Notably, OCN expression and calcification areas were increased in the induction group, and markedly promoted by IL-17A addition ( $\mathrm{P}<0.05$; Fig. 3A).

AKT2 signaling is involved in IL-17A induced early osteogenesis. IL-17A increased ALP and Runx-2 mRNA expression levels, particularly at 7 days, although these genes were downregulated following AKT2 knockdown $(\mathrm{P}<0.05$; Fig. $2 \mathrm{~A}$ and B). ALP protein levels were additionally detected at 7 days of incubation. Cells in the AKT2 knockdown and wild type groups exhibited markedly increased relative ALP activities in Ind. compared with NM. In addition, ALP activity was markedly increased by IL-17A $(\mathrm{P}<0.05)$, an effect which was inhibited following AKT2 knockdown ( $<<0.05$; Fig. 2C). IL-17A exerted no overt promotive effects on ALP activity 

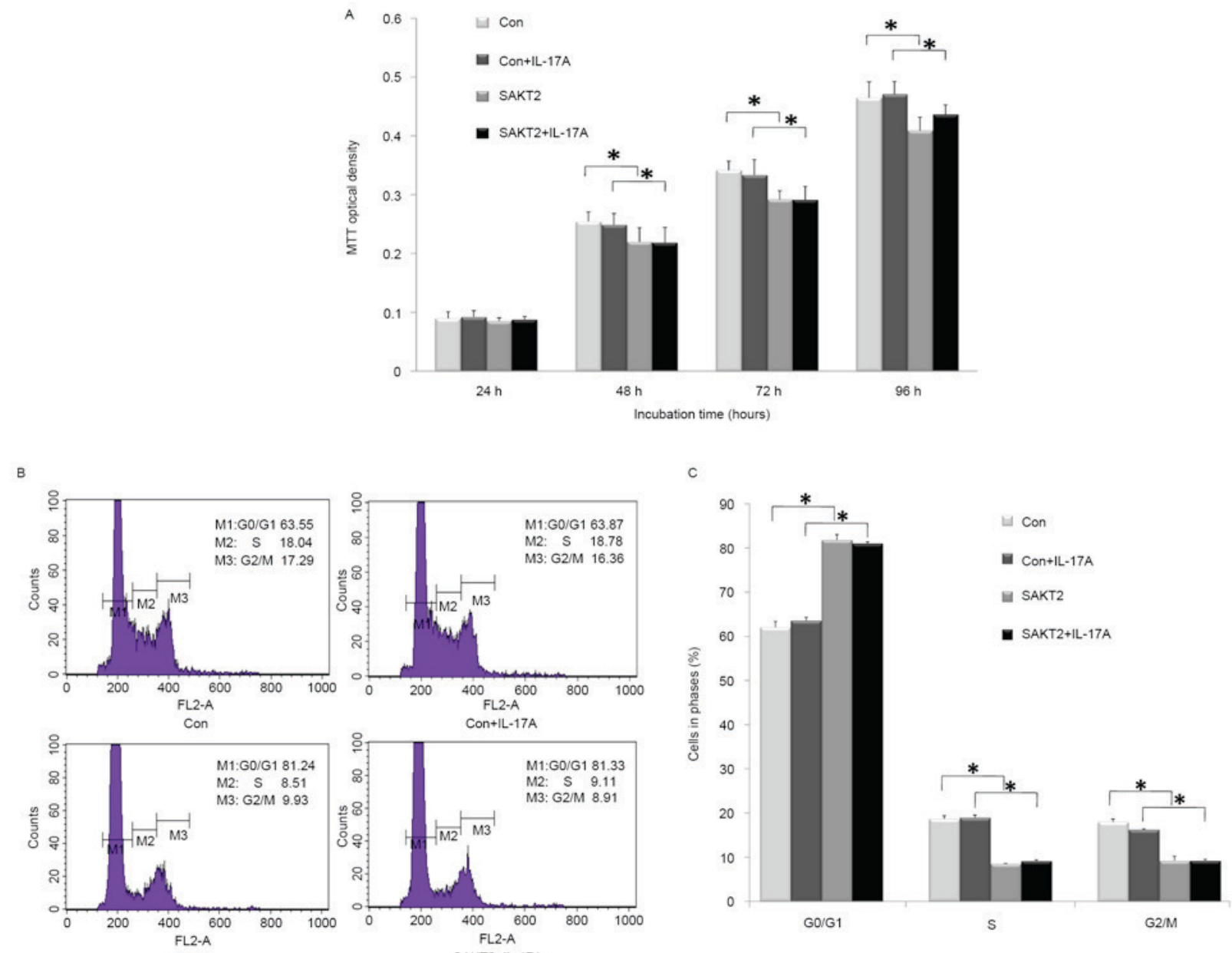

Figure 1. Effect of IL-17A and AKT2 on MC3T3-E1 cell proliferation. (A) MTT assay data demonstrating the proliferation of wild type and Akt2-- MC3T3-E1 cells incubated in normal medium with or without IL-17A $(50 \mathrm{ng} / \mathrm{ml})$ for $24,48,72$ and $96 \mathrm{~h}$. (B) Cells incubated for $48 \mathrm{~h}$ with or without IL-17A were assessed for cell cycle distribution by flow cytometry following propidium iodide staining. Representative flow cytograms are shown. (C) Quantitative analysis was performed on the flow cytometry data (mean \pm standard deviation). ${ }^{*} \mathrm{P}<0.05$. AKT2, RAC- $\beta$ serine/threonine protein kinase; SAKT2, Akt2 ${ }^{-c e l l s ; ~ I L-17 A, ~}$ interleukin-17A; Con, control.

with AKT2 knockdown (P>0.05; Fig. 2C). The results of the present study indicated that AKT2 signaling was required for the IL-17A-mediated promotion of osteogenic differentiation, while other signaling pathways may additionally be involved (Fig. 2).

AKT2 signaling is involved in IL-17A-induced late osteogenesis. The increased OCN gene expression induced by IL-17A was reduced by AKT2 knockdown $(\mathrm{P}<0.05$; Fig. 3A). As presented in Fig. 3B and $\mathrm{C}$, no calcification deposits were detected in cells cultured in NM alone, although they were observed in SAKT2 and control group cells cultured in Ind. The latter exhibited higher levels of calcification area, with a more pronounced increase upon IL-17A addition. However, AKT2 knockdown significantly weakened this effect $(\mathrm{P}<0.05)$. IL-17A exerted no overt calcification-promoting effects following AKT2 knockdown ( $\mathrm{P}>0.05)$.

Effects of IL-17A and AKT2 knockdown on IL-17RA mRNA expression in MC3T3-El cells. As presented in Fig. 4, IL-17RA mRNA was detected at baseline, and significantly increased following IL-17A stimulation $(\mathrm{P}<0.05)$. In addition, there was no significant difference in IL-17RA mRNA expression between AKT2 knockdown and wild type MC3T3-E1 cells when induced by IL-17A ( $\mathrm{P}>0.05)$.

Effects of IL-17A and AKT2 knockdown on p-PI3K and PI3K expression in MC3T3-El cells. To further investigate whether IL-17A-induced MC3T3-E1 cell alterations were mediated by the PI3K signaling pathway, the effect of IL-17A on p-PI3K and PI3K (p85) expression was assessed (Fig. 5). PI3K exhibited increased phosphorylation levels in Ind. compared with NM, in AKT2 knockdown and wild type cells $(\mathrm{P}<0.05$; Fig. 5B and D). However, p-PI3K amounts were significantly increased following treatment with IL-17A $(\mathrm{P}<0.05$; Fig. 5B and D); total PI3K and GAPDH were used as internal controls (Fig. 5).

\section{Discussion}

IL-17 regulates local inflammation in addition to osteoclastogenic mediators in gingival resident cells during the progression of periodontitis (4); indeed, the gingival concentration of IL-17 is above normal in severe attachment loss sites in 

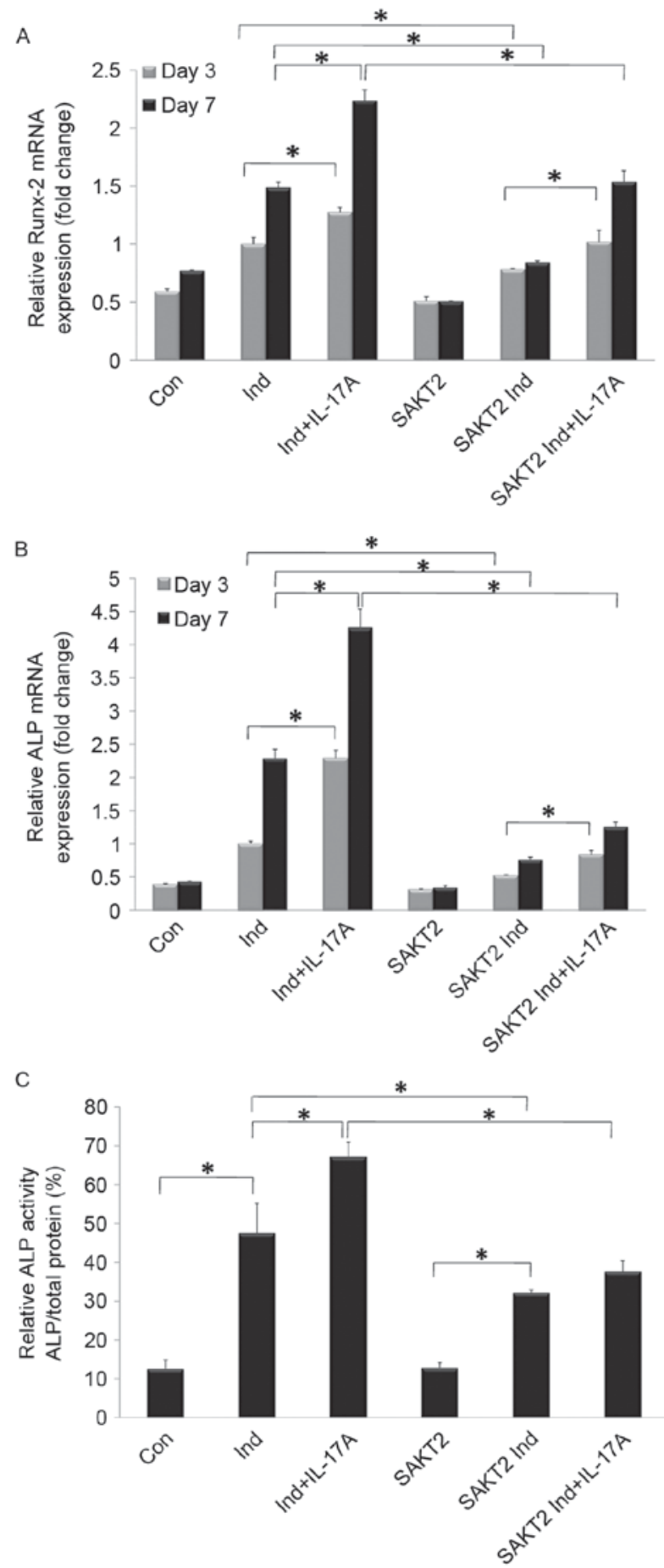

Figure 2. Effects of IL-17A on the expression levels of osteoblast-specific marker genes in cells during the early stages of osteogenic differentiation. Cells were harvested at 3 and 7 days for quantitative gene expression analysis of (A) Runx-2 and (B) ALP. Data were normalized to GAPDH expression and presented as a fold-change relative to the induction group at 3 days. (C) Effects of IL-17A on ALP activity at 7 days (mean \pm standard deviation). "P<0.05. IL-17A, interleukin-17A; Runx-2, Runt-related transcription factor 2; ALP, alkaline phosphatase; Con, control; Ind, osteogenic induction; AKT2, RAC- $\beta$ serine/threonine protein kinase; SAKT2, Akt2 ${ }^{-}$cells.

periodontitis (22), and IL-17A amounts are significantly higher in periodontal lesions adjacent to bone destruction (23). These findings indicated that IL-17 exhibits pleiotropic biological activities, and is associated with the severity of inflammation in periodontal lesions.
Additional insights into elucidating distinct functions for AKT2 have been proposed in cellular activity, demonstrating a significant role for AKT2 as a central signaling factor in the modulation of bone morphogenetic protein-stimulated osteogenic differentiation (12). Vascular smooth muscle cell differentiation appears to require the activation of AKT2 by PI3K (24). In a previous study, osteoclast bone resorption medium was demonstrated to promote differentiation and calcification in MC3T3-E1 cells, while AKT2-specific knockdown weakened the osteogenic effects (11). These previous findings provided a basis for understanding the importance of AKT2 signaling in osteoblast activity.

The functional association between bone cell proliferation and the initiation and progression of events associated with differentiation was proposed (25). Therefore, preosteoblastic MC3T3-E1 cell proliferation may support its osteogenic activity throughout the bone cellular metabolism process. Concomitantly, a number of downstream signaling pathways of PI3K affect cell growth and survival, including serine/threonine protein kinases (AKT and 3-phosphoinositide-dependent protein kinase 1) and protein tyrosine kinases (Tec family) (14). In this context, it was observed that cells lacking AKT2 exhibited growth deficiency, with slower proliferation. Additionally, AKT2 signaling regulates cancer cell growth and survival (13), and the suppression of PI3K/AKT signaling using specific inhibitors results in decreased osteoblast proliferation (26-28). Cell cycle distribution was assessed in the present study. Notably, AKT2 knockdown regulated cell proliferation by inducing cell cycle arrest in the G0/G1 phase and decreasing the number of cells in the $\mathrm{S}$ and $\mathrm{G} 2 / \mathrm{M}$ phases. The results of the present study provided additional evidence that PI3K/AKT activity is required during a number of phases of the cell cycle to allow for entry into mitosis (29). However, no apparent alteration was observed following treatment with IL-17A in cell proliferation or cell cycle distribution, in wild type and AKT2 knockdown cells. The precise function of IL-17A in cell growth and survival remain controversial. A previous study indicated that IL-17A stimulated cardiac fibroblast proliferation and migration via activation of certain pathways (30), while Nograles et al (31) observed no apparent effect of IL-17 on keratinocyte proliferation in vitro, indicating that this matter merits further investigation.

The expression levels of Runx-2 and ALP were assessed as specific early markers of osteogenic differentiation. OCN expression and extracellular matrix mineralization (Alizarin Red staining) occur during the late stages of osteogenesis. Runx-2, an important transcription factor of the Runx family, is expressed in the early stage of osteogenesis, and potently initiates the expression of major bone matrix protein genes (32). ALP, generated early, stimulates osteoblast precursor cell differentiation (20). OCN, a terminal osteoblast differentiation marker, is an osteoblast-secreted hormone regulating insulin secretion and sensitivity (33). The gene expression levels of ALP, Runx-2, and OCN, in addition to calcification areas, were increased in the induction group, and further enhanced by IL-17A, indicating that IL-17A promoted differentiation and calcification in MC3T3-E1 cells. Previous findings suggested that IL-17F, the amino acid sequence most homologous to IL-17A (both located on the 


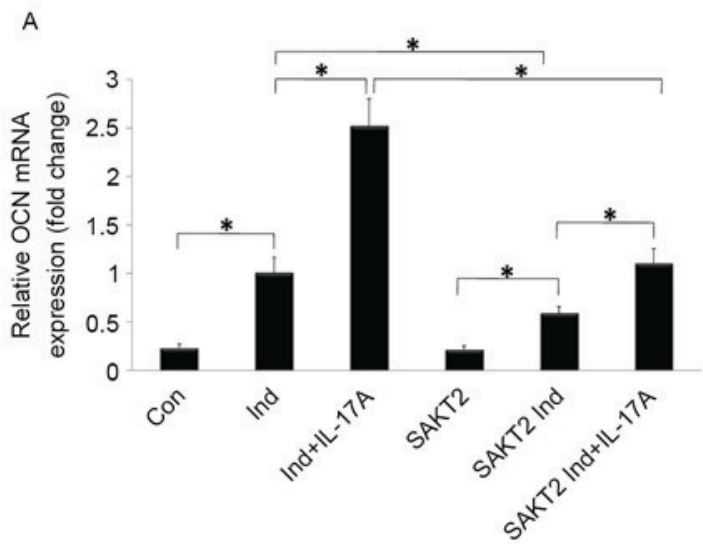

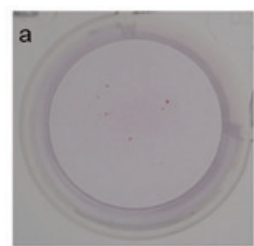
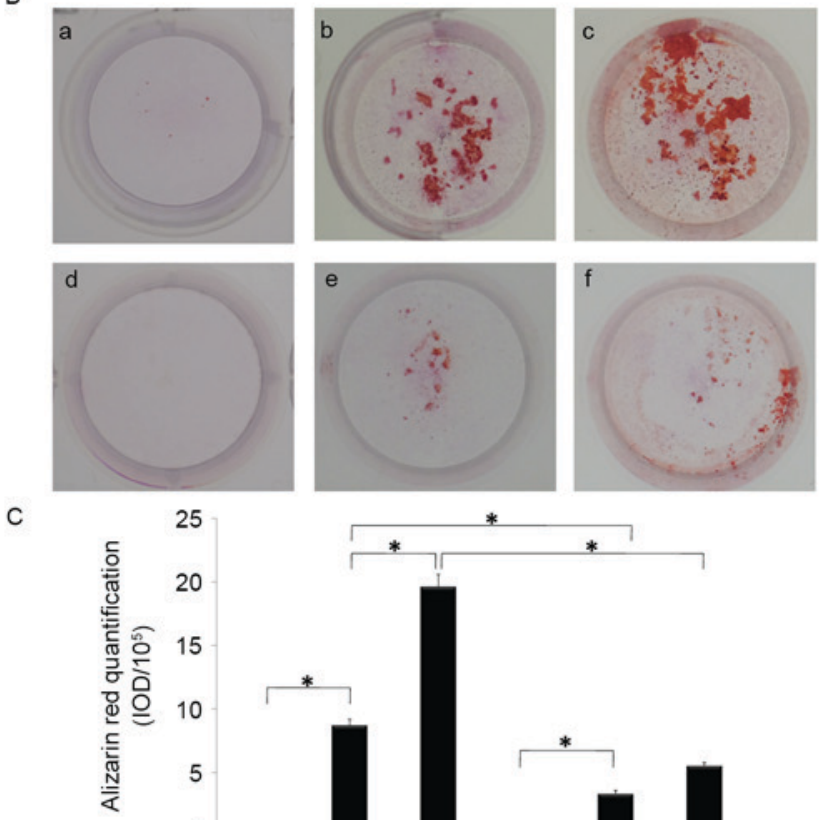
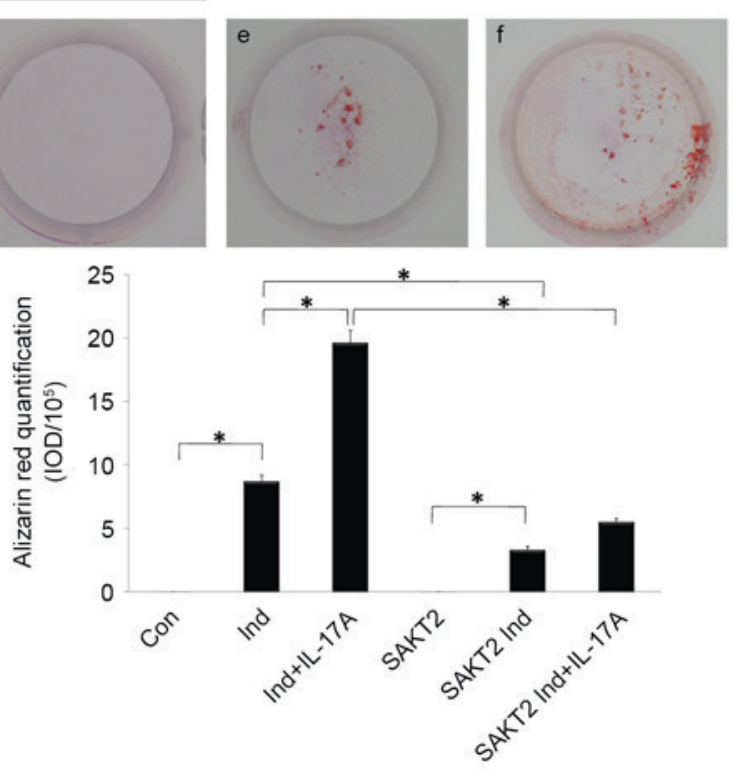

Figure 3. Effect of IL-17A and AKT2 on MC3T3-E1 cell calcification during the late osteogenesis stage. (A) IL-17A stimulated the expression of OCN, which was decreased by AKT2 knockdown. (B) Assessment of matrix mineralization of MC3T3-E1 cells by Alizarin Red S staining. Typical observations depicting mineralization. a, Con; b, Ind; c, Ind+IL-17A; d, SAKT2; e, SAKT2 Ind; f, SAKT2 Ind+IL-17A. (C) Quantitation of mineralization (mean \pm standard deviation) ${ }^{*} \mathrm{P}<0.05$. AKT2, RAC- $\beta$ serine/threonine protein kinase; SAKT2, Akt2 ${ }^{-}$cells; IL-17A, interleukin 17A; OCN, osteocalcin; Ind, osteogenic induction; Con, control.

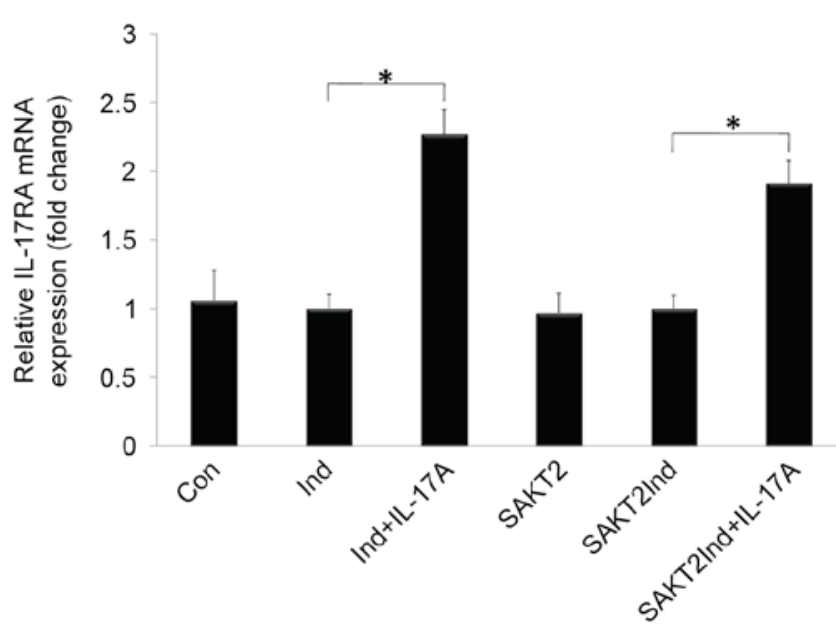

Figure 4. Effects of IL-17A on IL-17RA mRNA expression. IL-17RA mRNA expression was determined by reverse transcription-quantitative polymerase chain reaction analysis at 3 days post-stimulation with IL-17A. Data were normalized to GAPDH expression and presented as the fold-change relative to the induction group (mean \pm standard deviation). ${ }^{*} \mathrm{P}<0.05$. AKT2, RAC- $\beta$ serine/threonine protein kinase; SAKT2, Akt2 $\%$ cells; IL-17A, interleukin 17A; Ind, osteogenic induction; Con, control.

same chromosome) (34), directly rescued impaired healing and promoted bone synthesis in vitro in a model of early fracture repair (35), demonstrating a protective role in bone remodeling.
In the present study, the gene expression levels of Runx-2, ALP and OCN, in addition to relative ALP activity and calcification areas in AKT2 knockdown MC3T3-E1 cells, were reduced compared with values obtained for wild type cells under inducing conditions. Additionally, Runx-2 and ALP mRNA expression levels peaked at 7 days, with a time-dependent increase, corroborating findings by Luan et al (36). A specific role for AKT2 as a gatekeeper of osteogenic differentiation through regulation of Runx-2 gene expression was previously demonstrated (12); in addition, PI3K/AKT signaling is involved in Runx-2-dependent osteoblast differentiation, and the association between them is mutually dependent, further indicating that Runx-2 is involved in osteoblast differentiation (37). ALP and OCN regulation occurs at their promoter regions via interaction with Runx-2 (21). As early markers of osteoblast differentiation, these effectors may signal the beginning of matrix mineralization.

In addition, expression levels of osteoblast precursor cell differentiation markers at the early (Runx-2 and ALP) and late (OCN and Alizarin Red staining) stages in the control group were markedly increased following treatment with IL-17A, compared with values obtained for Akt $2^{-/-}$MC3T3-E1 cells under the same conditions, indicating that IL-17A promoted differentiation and calcification, while AKT2 signaling is required for such enhancement. However, compared with Akt2 ${ }^{-1} \mathrm{MC} 3 \mathrm{~T} 3-\mathrm{E} 1$ cells under osteogenic induction conditions, IL-17A continued to increase Runx-2, ALP and OCN mRNA 
A

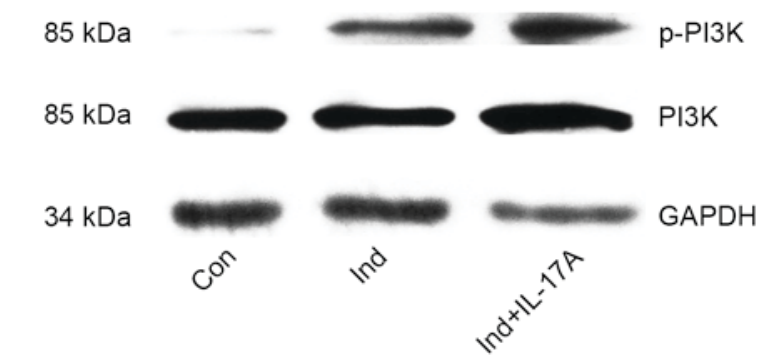

C

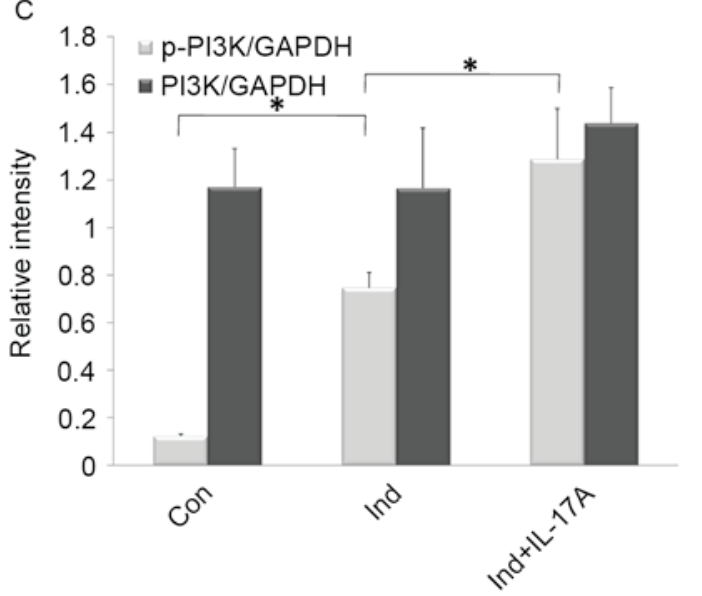

B

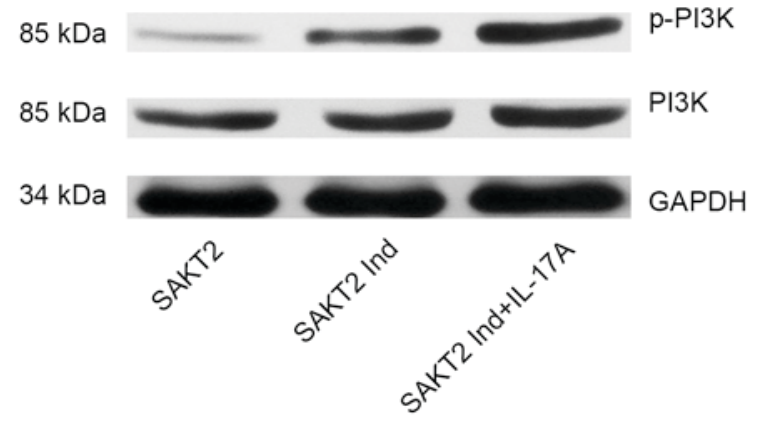

D

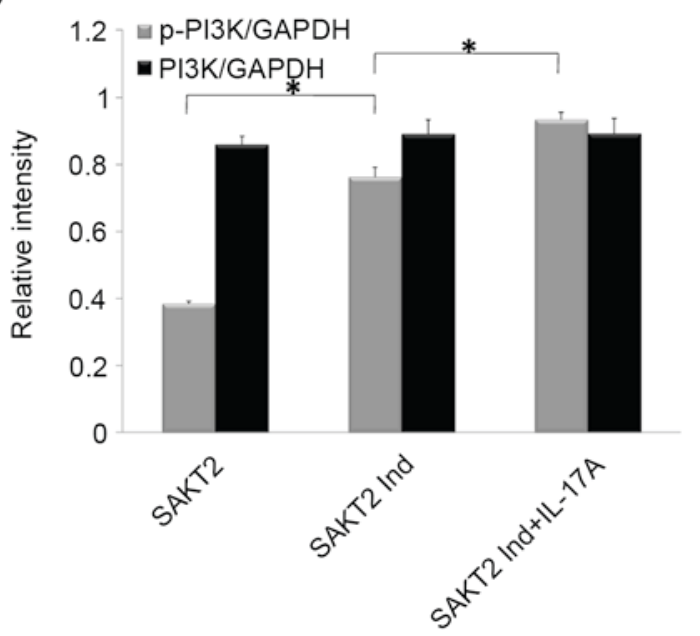

Figure 5. Effects of IL-17A on p-PI3K and PI3K expression in MC3T3-E1 cells. IL-17A increased the protein levels of p-PI3K in (A) wild type and (B) Akt2-1 MC3T3-E1 cells at $48 \mathrm{~h}$. (C) Densitometric analysis of p-PI3K and PI3K expression in wild type cells. (D) Densitometric analysis of p-PI3K and PI3K expression in Akt2 ${ }^{-/}$cells. "P<0.05. IL-17A, interleukin-17A; AKT2, RAC- $\beta$ serine/threonine protein kinase; SAKT2, Akt2 ${ }^{-/}$cells; PI3K, phosphatidylinositol 3-kinase; p, phosphorylated; Ind, osteogenic induction; Con, control.

expression levels, likely to be due to other signaling pathways which may participate in the IL-17A-mediated induction of osteogenic differentiation and calcification. Differences at the gene level were noted in Akt2 ${ }^{-/-}$MC3T3-E1 cells following IL-17A treatment, while the relative ALP activity level and calcification areas exhibited no alterations. However, significant differences were obtained for all these parameters between the control and IL-17A treatment groups. It is plausible that such effects are influenced by numerous factors and cellular signaling pathways, including the AKT2 signaling mentioned above and other pathways to be determined.

To clarify how IL-17A regulated osteoblast differentiation and calcification by AKT2 signaling, the present study identified osteoblastic MC3T3-E1 cells as target cells for IL-17A due to the increased expression of IL-17RA mRNA, corroborating van Bezooijen et al (10) who first described IL-17 as a new bone-acting cytokine in vitro. IL-17RA, the most highly-expressed member of the IL-17R family, was necessary for signal transduction mediated by IL-17A (38). However, no significant difference in IL-17RA mRNA expression was observed between AKT2 knockdown and wild type MC3T3-E1 cells when both induced by IL-17A, suggesting that AKT2 may lie downstream of IL-17RA, and there were no apparent variations in the expression of IL-17RA, even in the absences of AKT2. The results of the present study additionally demonstrated that IL-17A was able to upregulate
p-PI3K expression, consistent with others studies reporting that PI3K signaling serves an important role in bone development and growth $(12,39)$. These findings suggested that IL-17A is involved in regulating osteoblast activity. Therefore, IL-17A-induced differentiation and calcification in MC3T3-E1 cells may be mediated primarily through binding to IL-17RA, followed by the activation of PI3K and downstream AKT2 signaling.

In conclusion, the present study identified important roles for AKT2 in MC3T3-E1 cell proliferation and the cell cycle, additionally demonstrating that IL-17A promoted osteogenic differentiation and calcification, an effect involving AKT2 signaling. The results of the present study provided novel insights regarding the regulatory mechanism of IL-17A in alveolar bone remodeling, in addition to a basis for designing therapeutic targets for clinical periodontal disease. However, these preliminary data do not define accurately the complex association of bone remodeling with the immune system. Future investigations are required to examine how IL-17A regulates PI3K/AKT2 signaling to promote osteogenic differentiation and calcification, and whether other IL-17A-associated pathways, including mitogen-activated protein kinase and adapter protein CIKS/TNF receptor-associated factor 6 may be involved (38); further understanding of the biochemical and molecular mechanisms underlying 'osteoimmunology' are a prerequisite for periodontitis research. 


\section{Acknowledgements}

The present study was supported by the National Natural Science Foundation of China (grant nos. 81271142, 81400510 and 81300881), the Natural Science Foundation of Zhejiang Province (grant no. LY16H140002) and the Doctoral Program Foundation of the Ministry of Education of China (grant no. 20130101110011).

\section{References}

1. Pihlstrom BL, Michalowicz BS and Johnson NW: Periodontal diseases. Lancet 366: 1809-1820, 2005.

2. Bartold PM, Cantley MD and Haynes DR: Mechanisms and control of pathologic bone loss in periodontitis. Periodontology 53 55-69, 2000.

3. Arron JR and Choi Y: Bone versus immune system. Nature 408: 535-536, 2000.

4. Cheng WC, Hughes FJ and Taams LS: The presence, function and regulation of IL-17 and Th17 cells in periodontitis. J Clin Periodontol 41: 541-549, 2014.

5. Chen XT, Tan JY, Lei LH and Chen LL: Cytokine levels in plasma and gingival crevicular fluid in chronic periodontitis. Am J Dent 28: 9-12, 2015.

6. Chen XT, Chen LL, Tan JY, Shi DH, Ke T and Lei LH: Th17 and Th1 lymphocytes are correlated with chronic periodontitis. Immunol Invest 45: 243-254, 2016.

7. da Costa TA, Silva MJ, Alves PM, Chica JE, Barcelos EZ, Giani MA, Garlet GP, da Silva JS, Rodrigues Júnior V, Rodrigues DB and Cardoso CR: Inflammation biomarkers of advanced disease in nongingival tissues of chronic periodontitis patients. Mediators Inflamm 2015: 983782, 2015.

8. Yu JJ, Ruddy MJ, Wong GC, Sfintescu C, Baker PJ, Smith JB, Evans RT and Gaffen SL: An essential role for IL-17 in preventing pathogen-initiated bone destruction: Recruitment of neutrophils to inflamed bone requires IL-17 receptor-dependent signals. Blood 109: 3794-3802, 2007.

9. Settem RP, Honma K, Nakajima T, Phansopa C, Roy S, Stafford GP and Sharma A: A bacterial glycan core linked to surface (S)-layer proteins modulates host immunity through Th17 suppression. Mucosal Immunol 6: 415-426, 2013.

10. Van Bezooijen RL, Farih-Sips HC, Papapoulos SE and Löwik CW: Interleukin-17: A new bone acting cytokine in vitro. J Bone Miner Res 14: 1513-1521, 1999.

11. Chen LL, Huang M, Tan JY, Chen XT, Lei LH, Wu YM and Zhang DY: PI3K/AKT pathway involvement in the osteogenic effects of osteoclast culture supernatants on preosteoblast cells. Tissue Eng Part A 19: 2226-2232, 2013.

12. Mukherjee A, Wilson EM and Rotwein P: Selective signaling by Akt2 promotes bone morphogenetic protein 2-mediated osteoblast differentiation. Mol Cell Biol 30: 1018-1027, 2010.

13. Vivanco I and Sawyers CL: The phosphatidylinositol 3-Kinase AKT pathway in human cancer. Nat Rev Cancer 2: 489-501, 2002.

14. Cantley LC: The phosphoinositide 3-kinase pathway. Science 296: 1655-1657, 2002.

15. Melton AC, Melrose J, Alajoki L, Privat S, Cho H, Brown N, Plavec AM, Nguyen D, Johnston ED, Yang J, et al: Regulation of IL-17A production is distinct from IL-17F in a primary human cell co-culture model of T cell-mediated B cell activation. PLoS One 8: e58966, 2013.

16. Webster KE, Kim HO, Kyparissoudis K, Corpuz TM, Pinget GV, Uldrich AP, Brink R, Belz GT, Cho JH, Godfrey DI and Sprent J: IL-17-producing NKT cells depend exclusively on IL-7 for homeostasis and survival. Mucosal Immunol 7: 1058-1067, 2014.

17. Park YD, Kim YS, Jung YM, Lee SI, Lee YM, Bang JB and Kim EC: Porphyromonas gingivalis lipopolysaccharide regulates interleukin (IL)-17 and IL-23 expression via SIRT1 modulation in human periodontal ligament cells. Cytokine 60: 284-293, 2012.

18. Sudo H, Kodama HA, Amagai Y, Yamamoto S and Kasai S: In vitro differentiation and calcification in a new clonal osteogenic cell line derived from newborn mouse calvaria. J Cell Biol 96: 191-198, 1983.

19. Livak KJ and Schmittgen TD: Analysis of relative gene expression data using real-time quantitative PCR and the 2(-Delta Delta C(T)) method. Methods 25: 402-408, 2001.
20. Wang D, Christensen K, Chawla K, Xiao G, Krebsbach PH and Franceschi RT: Isolation and characterization of MC3T3-E1 preosteoblast subclones with distinct in vitro and in vivo differentiation/mineralization potential. J Bone Miner Res 14: 893-903, 1999.

21. Jeon EJ, Lee KY, Choi NS, Lee MH, Kim HN, Jin YH, Ryoo HM, Choi JY, Yoshida M, Nishino N, et al: Bone morphogenetic protein-2 stimulates Runx2 acetylation. J Biol Chem 281: 16502-16511, 2006.

22. Lester SR, Bain JL, Johnson RB and Serio FG: Gingival concentrations of interleukin-23 and -17 at healthy sites and at sites of clinical attachment loss. J Periodontol 78: 1545-1550, 2007.

23. Ohyama H, Kato-Kogoe N, Kuhara A, Nishimura F, Nakasho K, Yamanegi K, Yamada N, Hata M, Yamane J and Terada N: The involvement of IL-23 and the Th17 pathway in periodontitis. J Dent Res 88: 633-638, 2009.

24. Martin KA, Merenick BL, Ding M, Fetalvero KM, Rzucidlo EM, Kozul CD, Brown DJ, Chiu HY, Shyu M, Drapeau BL, et al: Rapamycin promotes vascular smooth muscle cell differentiation through insulin receptor substrate-1/phosphatidylinositol 3-kinase/Akt 2 feedback signaling. J Biol Chem 282: 36112-36120, 2007.

25. Stein GS and Lian JB: Molecular mechanisms mediating proliferation/differentiation interrelationships during progressive development of the osteoblast phenotype. Endocr Rev 14: 424-442, 1993.

26. Vinals F, Lopez-Rovira T, Rosa JL and Ventura F: Inhibition of $\mathrm{PI} 3 \mathrm{~K} / \mathrm{p} 70 \mathrm{~S} 6 \mathrm{~K}$ and p38 MAPK cascades increases osteoblastic differentiation induced by BMP-2. FEBS Lett 510: 99-104, 2002.

27. Martin SK, Fitter S, Bong LF, Drew JJ, Gronthos S, Shepherd PR and Zannettino AC: NVP-BEZ235, a dual pan class I PI3 Kinase and $\mathrm{mTOR}$ inhibitor, promotes osteogenic differentiation in human mesenchymal stromal cells. J Bone Miner Res 25: 2126-2137, 2010

28. Gu YX, Du J, Si MS, Mo JJ, Qiao SC and Lai HC: The roles of PI3K/Akt signaling pathway in regulating MC3T3-E1 preosteoblast proliferation and differentiation on SLA and SLActive titanium surfaces. J Biomed Mater Res A 101: 748-754, 2013.

29. Dangi S, Cha H and Shapiro P: Requirement for phosphatidylinositol-3 kinase activity during progression through S-phase and entry into mitosis. Cell Signal 15: 667-675, 2003.

30. Valente AJ, Yoshida T, Gardner JD, Somanna N, Delafontaine P and Chandrasekar B: Interleukin-17A stimulates cardiac fibroblast proliferation and migration via negative regulation of the dual-specificity phosphatase MKP-1/DUSP-1. Cell Signal 24: $560-568,2012$.

31. Nograles KE, Zaba LC, Guttman-Yassky E, Fuentes-Duculan J, Suárez-Fariñas M, Cardinale I, Khatcherian A, Gonzalez J, Pierson KC, White TR, et al: Th17 cytokines interleukin (IL)-17 and IL-22 modulate distinct inflammatory and keratinocyte-response pathways. Br J Dermatol 159: 1092-1102, 2008.

32. Komori T: Runx2, a multifunctional transcription factor in skeletal development. J Cell Biochem 87: 1-8, 2002.

33. Mundy GR and Elefteriou F: Boning up on ephrin signaling. Cell 126: 441-443, 2006.

34. Dong C: Diversification of T-helper-cell lineages: Finding the family root of IL-17-producing cells. Nat Rev Immunol 6: 329-333, 2006.

35. Nam D, Mau E, Wang Y, Wright D, Silkstone D, Whetstone H, Whyne C and Alman B: T-lymphocytes enable osteoblast maturation via IL-17F during the early phase of fracture repair. PLoS One 7: e40044, 2012.

36. Luan J, Cui Y, Zhang Y, Zhou X, Zhang G and Han J: Effect of CXCR4 inhibitor AMD3100 on alkaline phosphatase activity and mineralization in osteoblastic MC3T3-E1 cells. Biosci Trends 6: 63-69, 2012.

37. Fujita T, Azuma Y, Fukuyama R, Hattori Y, Yoshida C, Koida M, Ogita $\mathrm{K}$ and Komori T: Runx2 induces osteoblast and chondrocyte differentiation and enhances their migration by coupling with PI3K-Akt signaling. J Cell Biol 166: 85-95, 2004.

38. Gaffen SL: Structure and signalling in the IL-17 receptor family. Nat Rev Immunol 9: 556-567, 2009.

39. Ghosh-Choudhury N, Abboud SL, Nishimura R, Celeste A, Mahimainathan L and Choudhury GG: Requirement of BMP-2-induced phosphatidylinositol 3-kinase and Akt serine/threonine kinase in osteoblast differentiation and Smad-dependent BMP-2 gene transcription. J Biol Chem 277: 33361-33368, 2002. 